\title{
The effects of non-linear electron-phonon interactions on superconductivity and charge-density-wave correlations
}

\author{
Shaozhi Li and S. Johnston ${ }^{1}$ \\ ${ }^{1}$ Department of Physics and Astronomy, The University of Tennessee, Knoxville, TN 37996
}

(Dated: October 11, 2018)

\begin{abstract}
Determinant quantum Monte Carlo (DQMC) simulations are used to study non-linear electronphonon interactions in a two-dimensional Holstein-like model on a square lattice. We examine the impact of non-linear electron-lattice interactions on superconductivity and on Peierls charge-densitywave (CDW) correlations at finite temperatures and carrier concentrations. We find that the CDW correlations are dramatically suppressed with the inclusion of even a small non-linear interaction. Conversely, the effect of the non-linearity on superconductivity is found to be less dramatic at high temperatures; however, we find evidence that the non-linearity is ultimately detrimental to superconductivity. These effects are attributed to the combined hardening of the phonon frequency and a renormalization of the effective linear electron-phonon coupling towards weaker values. These results demonstrate the importance of non-linear interactions at finite carrier concentrations when one is addressing CDW and superconducting order and have implications for experiments that drive the lattice far from equilibrium.
\end{abstract}

PACS numbers: 02.70.Ss, 74.25.Kc, 71.38.-k

Electron-phonon (e-ph) coupling is an important interaction in many molecular systems and solids, which dresses carriers to form quasiparticles (called polarons) with increased effective masses and modified dispersion relations [1, 2]. Nearly all treatments of this interaction make use of linear models, where an electronic degree of freedom is coupled to the first order displacement of the ions. Theorists usually justify this by expanding the interaction in powers of the atomic displacement and then truncating the expansion to first order by assuming that the net displacements are small. But large displacements are expected in a number of situations. For example, in the limit of strong $e$-ph coupling, linear models predict large lattice distortions surrounding carriers as small polarons are formed [2 [5]. This result violates the assumptions underlying the linear models and indicates the necessity of including higher order terms in the expansion [4, 5].

Non-linear $e$-ph interactions have a dramatic effect on polaron properties in the single carrier limit. This was first demonstrated in Ref. 4, which examined the problem using the non-perturbative "momentum average" approximation and found that small higher-order interactions lead to a dramatic undressing of the polaron. This result is of potential relevance to many systems where strong $e$-ph interactions have been inferred from experiments [6 11]; however, it is not clear whether the single polaron result will generalize straightforwardly to the finite carrier concentrations relevant for these materials. Calculations at finite carrier concentrations are also needed in order to understand the impact of non-linearity on broken symmetry states like superconductivity [12] and charge-density-waves (CDW) 13.

Non-linearity can also be important when the $e$-ph interaction is weak. In some systems small initial atomic displacements can be driven far from equilibrium by an external potential. For instance, coherent phonon excitations have been observed in several pump-probe experiments [14 18. These experiments offer a promising new path to probing $e$-ph interactions, particularly in systems where the phonons have been difficult to differentiate from other collective excitations [19]. If the pump fluence is too high, however, it is possible that the lattice will be driven far from equilibrium, making higher-order terms in the $e$-ph interaction relevant.

In this letter we examine non-linear interactions in the many-body limit by studying the non-linear singleband Holstein model in two dimensions using determinant quantum Monte Carlo (DQMC). DQMC is a nonperturbative auxiliary-field technique capable of handling the $e$-ph interactions in a numerically exact fashion 20, 21. The technique is formulated in the grand canonical ensemble, which allows us to study the model at finite carrier concentrations and temperatures. DQMC has previously been applied to linear Holstein models 22 24, but to the best of our knowledge, it has not been applied to any $e$-ph models with non-linear interactions. Here we focus on the competition between Peierls CDW correlations and $s$-wave superconductivity that is known to occur in the linear model 22, 25]. As with the single carrier limit, we find that small non-linear interactions alter the properties of the system at finite carrier concentrations, undressing the carriers and significantly suppressing CDW correlations. While this allows superconductivity to emerge from behind the competing CDW order, we conclude that a non-linear interaction is ultimately detrimental to superconductivity in the Holstein model due to a renormalization of the effective linear $e$-ph coupling.

We consider a modified single-band Holstein Hamilto- 
nian $H=H_{\text {el }}+H_{\text {lat }}+H_{\text {int }}$, where

$$
\begin{gathered}
H_{\mathrm{el}}=-t \sum_{\langle i, j\rangle, \sigma} c_{i, \sigma}^{\dagger} c_{j, \sigma}-\mu \sum_{i, \sigma} \hat{n}_{i, \sigma} \\
H_{\text {lat }}=\sum_{i}\left(\frac{1}{2 M} \hat{P}_{i}^{2}+\frac{M \Omega^{2}}{2} \hat{X}_{i}^{2}\right)=\sum_{i} \Omega\left(b_{i}^{\dagger} b_{i}+\frac{1}{2}\right) \\
H_{\text {int }}=\sum_{i, \sigma, k} \alpha_{k} \hat{n}_{i, \sigma} \hat{X}_{i}^{k}=\sum_{i, \sigma, k} g_{k} \hat{n}_{i, \sigma}\left(b_{i}^{\dagger}+b_{i}\right)^{k}
\end{gathered}
$$

Here, $c_{i, \sigma}^{\dagger}\left(c_{i, \sigma}\right)$ creates (annihilates) an electron of spin $\sigma$ on lattice site $i ; b_{i}^{\dagger}\left(b_{i}\right)$ creates (annihilates) a phonon on site $i ; \hat{n}_{i, \sigma}=c_{i, \sigma}^{\dagger} c_{i, \sigma}$ is the electron number operator; $t$ is the nearest-neighbor hopping integral; $\mu$ is the chemical potential which sets the band filling; $\hat{X}_{i}$ and $\hat{P}_{i}$ are the position and momentum operators, respectively, for lattice site $i ; \Omega$ is the phonon frequency; $\langle\ldots\rangle$ denotes a sum over nearest neighbors; and $g_{k}=\alpha_{k}(2 M \Omega)^{-\frac{k}{2}}$ is the strength of the $e$-ph coupling to $k$-th order in displacement. Throughout this work consider a twodimensional square lattice with lattice spacing $a$ and set $a=M=t=1$ as the units of length, mass, and energy, respectively.

It is convenient to define a dimensionless $e$-ph coupling strength $\lambda$, which is given by the ratio of the lattice deformation energy $E_{p}$ to half the electronic bandwidth $W / 2$. For the linear Holstein Hamiltonian in two-dimensions $\lambda=\alpha_{1}^{2} /\left(M \Omega^{2} W\right)=g_{1}^{2} / 4 t \Omega$. For the non-linear model, additional dimensionless ratios $\xi_{n}=g_{n} / g_{n-1}$ must also be specified in order characterize the strength of the nonlinear terms. In the single carrier limit the quartic terms have a much weaker effect on the properties of the polaron in comparison to the quadratic terms [4]. We expect a similar result here. We therefore limit ourselves to the linear and quadratic order couplings only and define $\xi=g_{2} / g_{1}$. We note that one might be tempted to return to the physical definition of $\lambda$ in order to characterize the system with a single effective parameter. The implicit assumption here is that the system can be mapped onto an effective linear model with a renormalized phonon frequency $\Omega$ and $e$-ph coupling $g$. This, however, is not possible for the single particle case [4, where such effective linear models fail to capture the results of the nonlinear model. Therefore multiple parameters are needed to characterize the non-linear model. In order to facilitate easy comparisons with the linear case we keep the standard definition of $\lambda$, where $\lambda>1$ implies strong linear coupling, and use the ratio $\xi$ to characterize the strength of the non-linearity. Other choices are possible.

We study the non-linear model using DQMC. The method is outlined in a number of references [20, 21, and complete details of its application to the lattice degrees of freedom can be found in Ref. 24. The only change
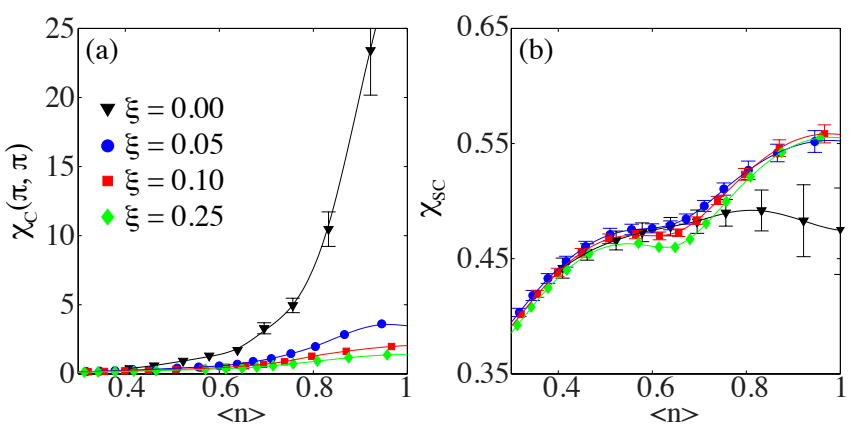

FIG. 1: (color online) (a) $\mathbf{q}=(\pi, \pi)$ charge and (b) pairfield susceptibilities as a function of filling for the linear $(\xi=$ 0 , black downward triangle) and non-linear $(\xi=0.05$, blue circle; $\xi=0.10$, red square; and $\xi=0.25$, green diamond) Holstein models with $\Omega=t$ and $\lambda=0.25$. The remaining parameters are $N=8 \times 8, \beta=5 / t$, and $\Delta \tau=0.1 / t$. Error bars smaller than the marker size have been suppressed for clarity.

from the procedure outlined therein is with regards to the definition of the $B$-matrices, which are defined on each discrete time slice $l$ [Eq. (11) of Ref. 24]; they must be modified to include the higher order interaction terms. Specifically, $B_{\sigma}(l)=\exp (-\Delta \tau v(l)) \exp (-\Delta \tau K)$, where $K$ is the matrix representation of $H_{\mathrm{el}}$ and $v(l)$ is a diagonal matrix whose $i$-th element is $[v(l)]_{i i}=\left(\sum_{k} \alpha_{k} X_{i, l}^{k}\right)$. All other aspects of the problem, including the sampling of the phonon fields, are treated as described in Ref. 24. Throughout this work we performed simulations with $\Delta \tau=1 / 10$ and generally work with clusters $N=8 \times 8$ in size, but some results are shown for other cluster sizes. We have examined clusters with linear dimensions ranging from $N=4$ to 12 and various values of $\Delta \tau$ for the half-filled case and found no significant finite size effects or $\Delta \tau$ errors. Finally, we note that both the linear and non-linear Holstein models do not exhibit a Fermion sign problem, which allows us to perform simulations to low temperatures.

We first examine the CDW and superconducting correlations. A measure of the CDW correlations is obtained from the charge susceptibility

$$
\chi_{C}(\mathbf{q})=\frac{1}{N} \int_{0}^{\beta} d \tau\left\langle\rho(\mathbf{q}, \tau) \rho^{\dagger}(\mathbf{q}, 0)\right\rangle,
$$

where $\rho(\mathbf{q})=\sum_{i, \sigma} e^{i \mathbf{q} \cdot \mathbf{R}_{i}} \hat{n}_{i, \sigma}$. Similarly, a measure of the $s$-wave superconducting correlations is obtained from the pair-field susceptibility

$$
\chi_{S C}=\frac{1}{N} \int_{0}^{\beta} d \tau\left\langle\Delta(\tau) \Delta^{\dagger}(0)\right\rangle,
$$

where $\Delta^{\dagger}=\sum_{\mathbf{k}} c_{\mathbf{k}, \uparrow}^{\dagger} c_{-\mathbf{k}, \downarrow}^{\dagger}=\sum_{i} c_{i, \uparrow}^{\dagger} c_{i, \downarrow}^{\dagger}$.

Fig. 1] shows the CDW and superconducting correlations as a function of the band filling in the linear and non-linear models at an inverse temperature $\beta=5 / t$. 
Here the linear coupling has been fixed to $\lambda=0.25$. The results for the linear model $(\xi=0)$ agree well with previous work [22, where $\mathbf{q}=(\pi, \pi)$ CDW correlations dominant due to a strong $\mathbf{q}=(\pi, \pi)$ nesting condition on the Fermi surface near half-filling $(\langle n\rangle \sim 1)$. 22, 25] The non-linear interaction dramatically alters these results. The initial effect is rapid and we find that $\chi_{C}(\pi, \pi)$ is suppressed near $\langle n\rangle \sim 1$ by an order of magnitude for a relatively small value of the non-linear coupling $\xi=0.05$. This suppression continues for increasing values of $\xi$, but it is less dramatic after the initial decrease.

The $\xi$ dependence of $\chi_{C}(\pi, \pi)$ and $\chi_{S C}$ is examined further in Fig. 2 Results are shown for a fixed filling of $\langle n\rangle=1$ and $\lambda=0.25$. The behavior matches the expectations from Fig. 1 and the rapid initial suppression of the CDW correlations for small non-zero values of $\xi$ is evident. Similar results were obtained in the single polaron limit, where a small value of $\xi$ produced large changes in the polaron's effective mass and quasiparticle weight, but gave way to more gradual changes in these properties for further increases in the value of $\xi$ 4. We also note that the results obtained for $N=8 \times 8$ and $N=10 \times 10$ clusters are nearly identical, indicating that the finite size effects are small and have little bearing on our conclusions.

In the linear model, CDW correlations directly compete with $s$-wave superconductivity and the former dominate at low temperatures, particularly for fillings near $\langle n\rangle \sim 1$ [22, 25]. Thus there is a concomitant enhancement in the pair-field susceptibility once the CDW correlations are suppressed by the non-linear interaction, which is evident in Figs. 1 and 2 After its initial rise, however, $\chi_{S C}$ is relatively independent of the value of $\xi$ for all values of the band filling examined, apart from a slight suppression of $\chi_{S C}$ in the vicinity of $\langle n\rangle \sim 0.65$. Thus the non-linear coupling does not significantly enhance or suppress superconductivity at this temperature once the competition with the CDW correlations has been suppressed or eliminated.

The inset of Fig. 2 plots the average lattice displacement $\langle X\rangle=\frac{1}{N} \sum_{i} X_{i}$ as a function of $\xi$. This quantity serves as a proxy for the average number of phonon quanta (which is not directly accessible in the DQMC formalism) as larger lattice distortions are described by coherent states with increasing numbers of phonon quanta. For increasing values of $\xi$, the average lattice displacement is reduced, and thus, so is the number of phonon quanta on each site. This is fully consistent with the single carrier limit where the number of phonon quanta in the polaron cloud dropped dramatically for non-zero values of $\xi$ 4. This relaxation of the lattice displacement shown here thus reflects the undressing of the lattice bipolarons that form the $\mathbf{q}=(\pi, \pi)$ CDW state.

The physical origin of the polaron undressing is the renormalization of the effective linear coupling by the non-linear interaction terms. This can be understood

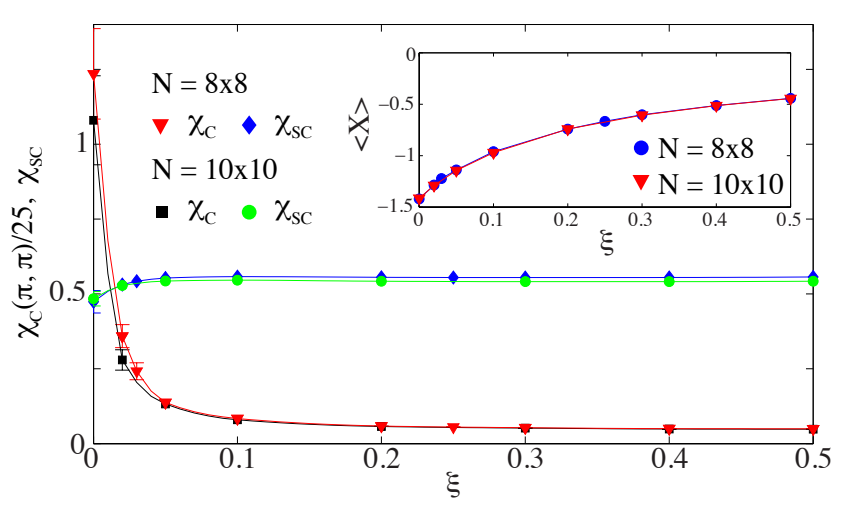

FIG. 2: (color online) The charge $\chi_{C}(\pi, \pi)$ (red downward triangle and blue diamond) and pair-field $\chi_{S C}$ (black square and green circle) susceptibilities as a function of $\xi$ at half filling $\langle n\rangle=1$. Results are shown for $N=8 \times 8$ and $10 \times 10$ clusters while the remaining parameters are the same as those used in Fig. 1. The charge susceptibility has been rescaled by a factor of 25 . Inset: The average value of the lattice displacement as a function of $\xi$ for both cluster sizes. Error bars smaller than the marker size have been suppressed for clarity.

from a mean-field treatment of the problem in the atomic limit 4]. Applying the mean-field decoupling $\left(b^{\dagger}\right)^{2}=$ $2 b^{\dagger}\left\langle b^{\dagger}\right\rangle-\left\langle b^{\dagger}\right\rangle^{2}$ leads to the effective Hamiltonian

$$
H_{M F}=\Omega_{M F}\left(b^{\dagger} b+\frac{1}{2}\right)+g_{M F} \hat{n}\left(b^{\dagger}+b\right)
$$

where $\Omega_{M F}=\Omega+2 g_{2}$ and $g_{M F}=g_{1}\left(1-\frac{2 g_{2}}{\Omega+4 g_{2}}\right)$. From this result one sees that the second order interaction acts to harden the phonon frequency and renormalize the effective linear coupling to lower values. This has the net effect of decreasing $\lambda_{M F} \sim \frac{g_{M F}^{2}}{\Omega_{M F}}$ and thus the effective $e$ ph interaction is weaker for the non-linear model. A similar effect occurs for the itinerant case examined here, as is evident from the relaxation of the average lattice displacement and the suppression of the CDW correlations. This observation also helps us understand why superconductivity is not strongly affected. While the hardening of the phonon frequency would raise the superconducting $T_{c}$, the net decrease in effective linear coupling results in an overall suppression of of the superconducting correlations and thus $T_{c}$.

If the non-linear coupling results in a undressing of the polarons via a weakening of the effective linear coupling, we would expect the system to relax back to a metallic state for large values of $\xi$. We therefore examine the spectral weight at the Fermi level order to confirm this expectation. A measure of the spectral weight at the Fermi level $(\omega=0)$ can be obtained from the imaginary 

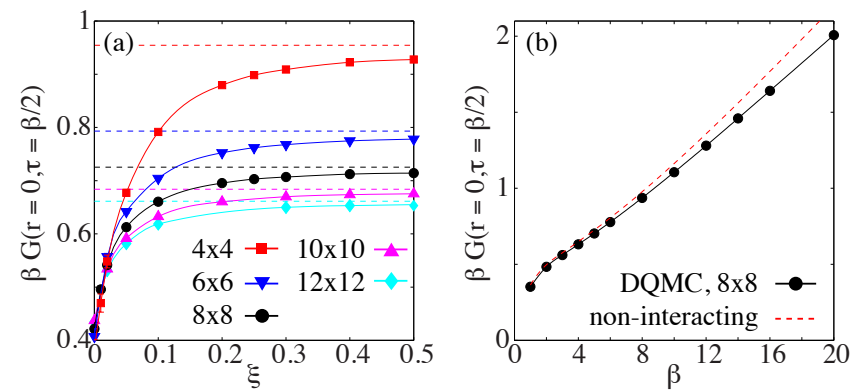

FIG. 3: (color online) (a) The spectral weight at the Fermi level given by $\beta G(\mathbf{r}=0, \tau=\beta / 2) \equiv \beta G_{\beta / 2}$ (see main text) as a function of the non-linear coupling $\xi$ for various cluster sizes, $\Omega=t$, and $\lambda=0.25$. The inverse temperature is $\beta=5 / t$. The dashed lines indicate the value for the non-interacting $(\lambda=\xi=0)$ case $\beta G_{\beta / 2}^{(0)}$. (b) The temperature dependence of $\beta G_{\beta / 2}$ for the $N=8 \times 8$ cluster and $\xi=0.25$. The red dashed line is the result for the non-interacting case. Error bars smaller than the marker size have been suppressed for clarity.

time Green's function via the relationship 26 .

$$
\beta G(\mathbf{k}, \tau=\beta / 2)=\frac{\beta}{2} \int d \omega A(\mathbf{k}, \omega) g(\omega, \beta),
$$

where $g(\omega, \beta)=\cosh ^{-1}(\beta \omega / 2)$ and $A(\mathbf{k}, \omega)=$ $-\frac{1}{\pi} \operatorname{Im} G(\mathbf{k}, \omega)$ is the spectral function. At low temperatures $g(\omega, \beta)$ is peaked at $\omega=0$ and therefore weights the spectral weight at the Fermi level. The local propagator $G\left(\mathbf{r}-\mathbf{r}^{\prime}, \tau=\beta / 2\right) \propto \sum_{\mathbf{k}} G(\mathbf{k}, \beta / 2)$ is then a measure of the total spectral weight at the Fermi level. For simplicity we introduce the notation $\beta G\left(\mathbf{r}=\mathbf{r}^{\prime}, \tau=\beta / 2\right) \equiv \beta G_{\beta / 2}$.

Fig. 3 a shows $\beta G_{\beta / 2}$ as a function of $\xi$. Results are shown at half-filling for a number of cluster sizes and the behavior is similar for all cases examined. For $\xi=0$ the CDW correlations dominate, resulting in the formation of a CDW gap that reduces the spectral weight at $\omega=0$. The spectral weight is restored for increasing values of $\xi$, which is consistent with the closing of the CDW gap. For large $\xi$ the value of $\beta G_{\beta / 2}$ approaches the non-interacting value, which is indicated by the dashed lines. Therefore, at $\beta=5 / t$, the system is metallic but with a slightly reduced $\xi$-dependent spectral weight. The metallicity of the system is further evidenced by the temperature dependence of $\beta G_{\beta / 2}$, shown in Fig. $3 \mathrm{~b}$ for the $\xi=0.25$, $N=8 \times 8$ case. Here $\beta G_{\beta / 2}$ increases for decreasing temperatures as expected for a Fermi liquid where the quasiparticle scattering rate scales as $T^{2}$. The full DQMC result, however, deviates from the non-interacting limit, indicating that the quasiparticles remain slightly dressed by the $e$-ph interaction. This picture is consistent with the one obtained from the single carrier limit, where the small polaron relaxes to a large polaron with a renormalization factor $Z$ only slightly reduced from 1 at large values of $\xi[4$.
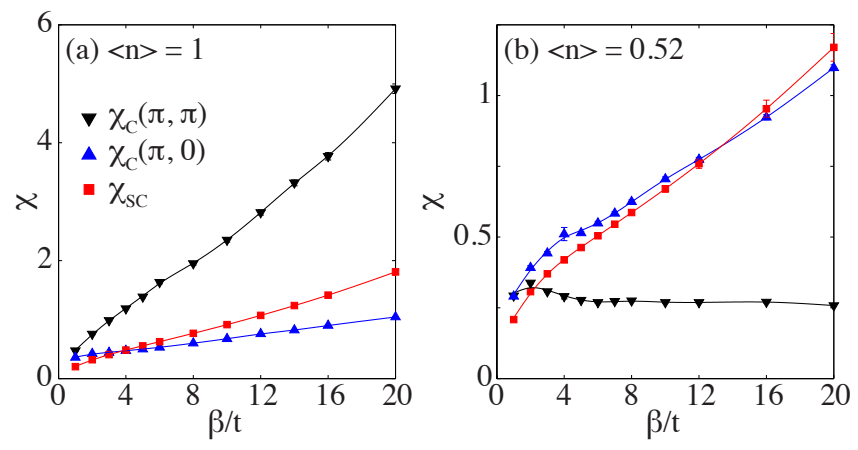

FIG. 4: (color online) The charge $\chi_{C}(\mathbf{q})$ and pair-field $\chi_{S C}$ susceptibilities as a function of the inverse temperature for the non-linear model $(\xi=0.25)$ at fillings (a) $\langle n\rangle=1$ and (b) $\langle n\rangle=0.52$. The charge susceptibilities at wavevectors $\mathbf{q}=(\pi, \pi)$ (black downward triangle) and $\mathbf{q}=(\pi, 0)$ (blue upward triangle) are shown. The remaining parameters were $\lambda=0.25, \Omega=t$, and $\Delta \tau=0.1 / t$. Error bars smaller than the marker size have been suppressed for clarity.

We have demonstrated that the system re-enters a metallic phase as the value of $\xi$ is increased and the effective linear coupling is decreased. We would therefore like to assess if superconductivity emerges as the ground state if the temperature is lowered further. To examine this, Fig. 4 a plots the temperature dependence of several relevant susceptibilities for the half- (Fig. 4a) and approximately quarter-filled models (Fig. 4p). At half-filling the $\mathbf{q}=(\pi, \pi)$ CDW correlations are weakened, however, they remain as the dominate correlations in the system for all values of $\xi$ examined [for reference, $\chi_{C}(\pi, \pi) / \chi_{S C} \sim 2.2$ for $\xi=0.5$ and $\left.\beta=5 / t\right]$. This remains true upon further cooling and thus the ground state of the system with $\xi=0.25$ remains a $\mathbf{q}=(\pi, \pi)$ CDW insulator albeit with a drastically reduced transition temperature.

Away from half-filling $\chi_{C}(\pi, \pi)$ is reduced by a combination of the non-linear interaction and the loss of the Fermi surface nesting at this wavevector. For example, in the vicinity of a quarter filling we find $\chi_{S C}>\chi_{C}(\pi, \pi)$. But other ordering vectors become relevant at these fillings, and for $\langle n\rangle \sim 0.52$ we find that $\mathbf{q}=(\pi, 0)$ becomes the dominant ordering vector. Moreover, for $\beta=5 / t$, $\chi_{C}(\pi, 0) \sim \chi_{S C}$ suggesting that superconductivity could emerge as the ground state at this filling. For decreasing temperatures $\chi_{C}(\pi, 0)$ and $\chi_{S C}$ increase concurrently, but the superconducting pair-field susceptibility overtakes the charge susceptibility at $\beta \sim 14 / t$. This signals a superconducting ground state at low temperature but with a reduced $\mathrm{T}_{c}$ owing to the renormalized effective linear coupling.

Finally, in Fig 5 we examine the dependence of our results at half-filling on the linear coupling strength $\lambda$ and the phonon frequency $\Omega$. Figs. $5 \mathrm{a}$ and $5 \mathrm{~b}$ show 

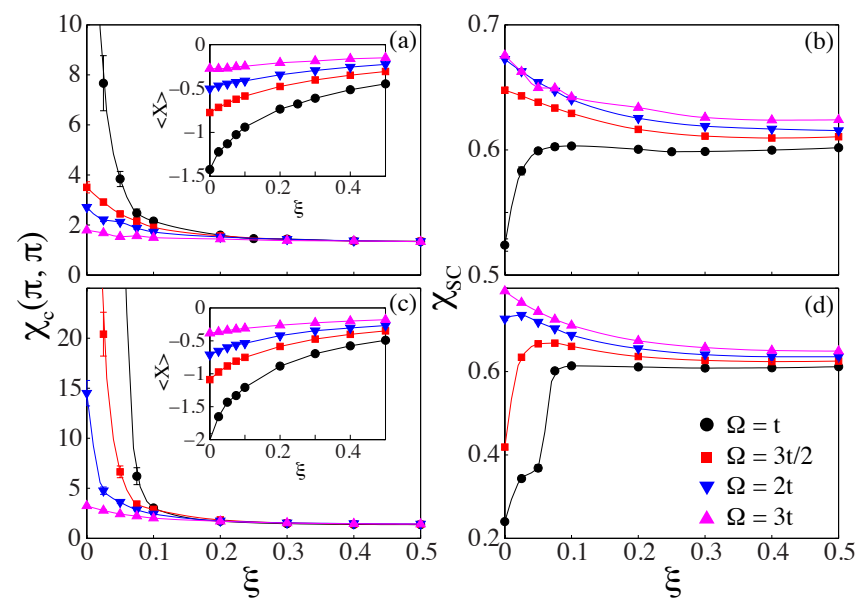

FIG. 5: (color online) The $\Omega$-dependence of the (a), (c) charge $\chi_{C}(\pi, \pi)$ and (b), (d) superconducting pair field $\chi_{S C}$ susceptibilities for the half-filled model. The linear couplings are $\lambda=0.25$ for the top panels and $\lambda=0.5$ for the bottom panels. Results are shown for an $N=8 \times 8$ cluster. Error bars smaller than the marker size have been suppressed for clarity.

$\chi_{C}(\pi, \pi)$ and $\chi_{S C}$, respectively, for $\Omega=t, 3 t / 2,2 t$, and $3 t$ and $\lambda=0.25$. Figs. 5 and $5 \mathrm{~d}$ show similar results for $\lambda=0.5$. The results follow the trends we have discussed. For $\xi=0$ the CDW correlations increase with increasing linear coupling or with decreasing phonon frequency, consistent with prior work [22]. In all cases, however, the CDW correlations are suppressed for increasing nonlinear interaction strengths. The weakening of the effective linear coupling and suppression of the CDW ordered phase by the non-linear interaction is therefore a generic result.

In summary, we have examined a non-linear Holstein model on a two-dimensional square lattice and at finite temperatures and carrier concentrations using determinant quantum Monte Carlo. The competition between CDW and superconducting correlations was re-examined as a function of the non-linear $e$-ph interaction strength. The primary effect of the non-linear coupling is a dramatic suppression of the CDW correlations that dominate the linear model. A less pronounced effect was observed for the superconducting correlations. These effects are attributed to a combined hardening of the phonon frequency and renormalization of the effective linear coupling by the non-linear terms. The net result is an overall reduction in the $e$-ph interaction strength. We therefore conclude that the non-linear interactions in the Holstein model will also reduce the transition temperature for phonon-mediated superconductivity. Our results explicitly confirm the importance of the non-linear interactions at finite carrier concentrations, as proposed in Ref. 4 and show that the undressing of the polaron by the non-linear interactions is generic.
Our results have implications for pump-probe experiments aimed at studying the strength of the $e$-ph interaction. For example, the higher order interaction terms can become important in such experiments if the external field drives the lattice to large displacements, even if the electron-lattice coupling at equilibrium is weak. Therefore, if the lattice is pumped too strongly there is a danger that the non-linearity will enter and renormalize the effective coupling to smaller values. In doing so, one could drive the system hard enough that they extinguish the very interactions they are trying to probe. Obviously this will be less of an issue if the pump pulses are weak and the lattice is only slightly perturbed; however, these effects may become extremely important if the lattice is strongly pumped, as our results show that even a small non-linear contribution can have an order-of-magnitude impact. Moving forward it will be important to study the role of non-linear electron-lattice coupling and anharmonic lattice potentials as the community continues to study systems driven far from equilibrium.

Finally, these results call for a re-evaluation of claims of high- $\mathrm{T}_{c}$ superconductivity mediated by non-linear $e$ ph coupling [27]. We stress, however, that physics arising from the non-linear coupling is different from anharmonic effects due to the lattice potential, which are thought to play a key role in $\mathrm{MgB}_{2}$ [10] and $\mathrm{KOs}_{2} \mathrm{O}_{6}$ [28].

We thank M. Berciu and D. J. Scalapino for useful discussions. This work is partially based upon computational resources supported by the University of Tennessee and Oak Ridge National Laboratorys Joint Institute for Computational Sciences (http://www.jics.utk.edu).

[1] S. Engelsberg and J. R. Schrieffer, Phys. Rev. 131, 993 (1963).

[2] J. T. Devreese, Encyclopedia of Applied Physics 14, 383 (1996).

[3] G. L. Goodvin, M. Berciu, and G. A. Sawatzky, Phys. Rev. B 74, 245104 (2006).

[4] C. P. J. Adolphs and M. Berciu, EPL 102, 47003 (2013).

[5] C. P. J. Adolphs and M. Berciu, Phys. Rev. B 90, 085149 (2014).

[6] A. Lanzara et. al., Nature 412, 510 (2001).

[7] W. S. Lee et. al., Phys. Rev. Lett. 110, 265502 (2013).

[8] N. Mannella et al., Nature, 438, 474 (2005).

[9] M. Medarde et al., Phys. Rev. Lett. 80, 2397 (1998).

[10] T. Yildirim et al., Phys. Rev. Lett. 87, 037001 (2001).

[11] S. Ciuchi and S. Fratini, Phys. Rev. Lett.106, 166403 (2011).

[12] B. Bardeen, J. Cooper, and J. R. Schrieffer, Phys. Rev. 108, 1175 (1957).

[13] G. Grüner, Rev. Mod. Phys. 60, 1129 (1988).

[14] L. Perfetti et al., Phys. Rev. Lett. 97, 067402 (2006).

[15] F. Schmitt et al., Science 321, 1649 (2008).

[16] A. D. Caviglia et al., Phys. Rev. Lett. 108, 136801 (2012).

[17] E. Papalazarou et al., Phys. Rev. Lett. 108, 256808 
(2012).

[18] L. X. Lang et al., Phys. Rev. Lett. 112, 207001 (2014).

[19] W. Zhang et al., Nat. Comm. 5, 4959 (2014).

[20] S. R. White et al., Phys. Rev. B 40, 506 (1989).

[21] R. Blankenbecler, D. J. Scalapino, and R. L. Sugar, Phys. Rev. D, 24, 2278 (1981).

[22] R. T. Scalettar, N. E. Bickers, and D. J. Scalapino, Phys. Rev. B, 40, 197 (1989).

[23] C. E. Creffield, G. Sangiovanni, and M. Capone, Eur.
Phys. J B 44, 175 (2005)

[24] S. Johnston et al., Phys. Rev. B 87, 235133 (2013).

[25] F. Marsiglio, Phys. Rev. B 42, 2416 (1990).

[26] N. Trivedi and M. Randeria, Phys. Rev. Lett. 75, 312 (1995).

[27] D. M. Newns and C. C. Tsuei, Nat. Phys. 3, 184 (2007),

[28] J. Chang, I. Eremin, and P. Thalmeier, New J. Phys. 11, 055068 (2009). 